\title{
A capillary specimen aberration for describing X-ray powder diffraction line profiles for convergent, divergent and parallel beam geometries
}

\author{
Alan A. Coelho and Matthew R. Rowles
}

J. Appl. Cryst. (2017). 50, 1331-1340

\section{IUCr Journals CRYSTALLOGRAPHY JOURNALS ONLINE \\ Copyright (C) International Union of Crystallography \\ Author(s) of this paper may load this reprint on their own web site or institutional repository provided that this cover page is retained. Republication of this article or its storage in electronic databases other than as specified above is not permitted without prior permission in writing from the IUCr. \\ For further information see http://journals.iucr.org/services/authorrights.html}


JOURNAL OF

APPLIED

CRYSTALLOGRAPHY

ISSN 1600-5767

Received 20 April 2017

Accepted 31 July 2017

Edited by S. Boutet, SLAC National Accelerator Laboratory, Menlo Park, USA

Keywords: cylindrical capillary specimens; X-ray diffraction; Rietveld refinement; fundamental parameters; TOPAS-Academic.

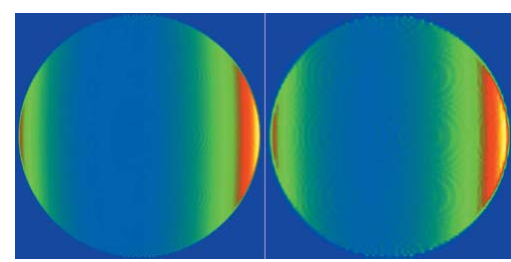

C 2017 International Union of Crystallography

\section{A capillary specimen aberration for describing $X$-ray powder diffraction line profiles for convergent, divergent and parallel beam geometries}

\author{
Alan A. Coelho ${ }^{\mathrm{a} *}$ and Matthew R. Rowles ${ }^{\mathrm{b}}$ \\ ${ }^{\mathbf{a}} 72$ Cedar Street, Wynnum, Brisbane, Queensland 4178, Australia, and ${ }^{\mathbf{b}}$ Department of Physics and Astronomy, Curtin \\ University, Australia. ${ }^{*}$ Correspondence e-mail: alancoelho@bigpond.com
}

X-ray powder diffraction patterns of cylindrical capillary specimens have substantially different peak positions, shapes and intensities relative to patterns from flat specimens. These aberrations vary in a complex manner with diffraction angle and instrument geometry. This paper describes a fast numerical procedure that accurately describes the capillary aberration in the equatorial plane for convergent focusing, divergent and parallel beam instrument geometries. Axial divergence effects are ignored and only a cross section of the capillary, a disc, is considered; it is assumed that axial divergence effects can be described using an additional correction that is independent of the disc correction. Significantly, the present implementation uses the TOPAS-Academic aberration approximation technique of averaging nearby aberrations in $2 \theta$ space to approximate in-between aberrations, which results in no more than $\sim 30$ disc aberrations calculated over the entire $2 \theta$ range, even when the diffraction pattern comprises thousands of peaks. Finally, the disc aberration is convoluted with the emission profile and other instrument and specimen aberrations in a Rietveld refinement sense, allowing for refinement on the specimen's absorption coefficient and capillary diameter, as well as the instrument focal length. Large differences between refined and expected values give insight into instrument alignment.

\section{Introduction}

Diffraction peak positions and intensities collected from a capillary specimen in parallel beam geometry are typically corrected using an analytical function (Sabine et al., 1998). Correcting for peak shapes, however, has not previously been performed, most probably because of its complexity and the estimated large computing power necessary. Nevertheless, with careful numerical procedures, the peak shapes can be accurately described with a small amount of computation. We first define the two instrument geometries of convergent and divergent beam geometries as seen in Fig. 1. Parallel beam geometry can be thought of as being a case of an infinite focal length. For the case of capillaries illuminated with an X-ray beam short in length in the axial plane, the specimen can be considered a disc centred on the diffractometer axis with consideration given only to the equatorial plane (Sulyanov et $a l ., 2012)$. It is assumed that the intensity distribution of the incident beam is uniform and that the entire disc is bathed in $\mathrm{X}$-rays. The peak shape aberration along the length of the capillary in the axial plane can then be further described using an axial divergence aberration (Cheary \& Coelho, 1998) and is not considered here.

The intensity contribution from the whole disc has been determined numerically by Dwiggins (1972, 1975); however, 
the objective here is not only to determine intensity contributions but to additionally determine the shape of the aberration. The formation of the disc aberration can be broken down into three steps (Fig. 1): (i) calculation of diffraction intensities along lines parallel to the diffracting vector (along the $x$ axis) for $x>0$ and separately for $x<0$; (ii) summing the line aberrations into a single aberration ensuring the centroid and intensity of the aberration are accurate; and (iii) applying a smoothing function to smooth out ripples caused by the discrete nature of the process. From symmetry, a line aberration calculated at $y$ is a good approximation to the aberration at $-y$, but shifted along the $2 \theta$ axis.

\section{Diffracting intensities from a line on the disc parallel to the $x$ axis}

The following development of the disc aberration applies to both convergent and divergent beam geometries (Fig. 1). In
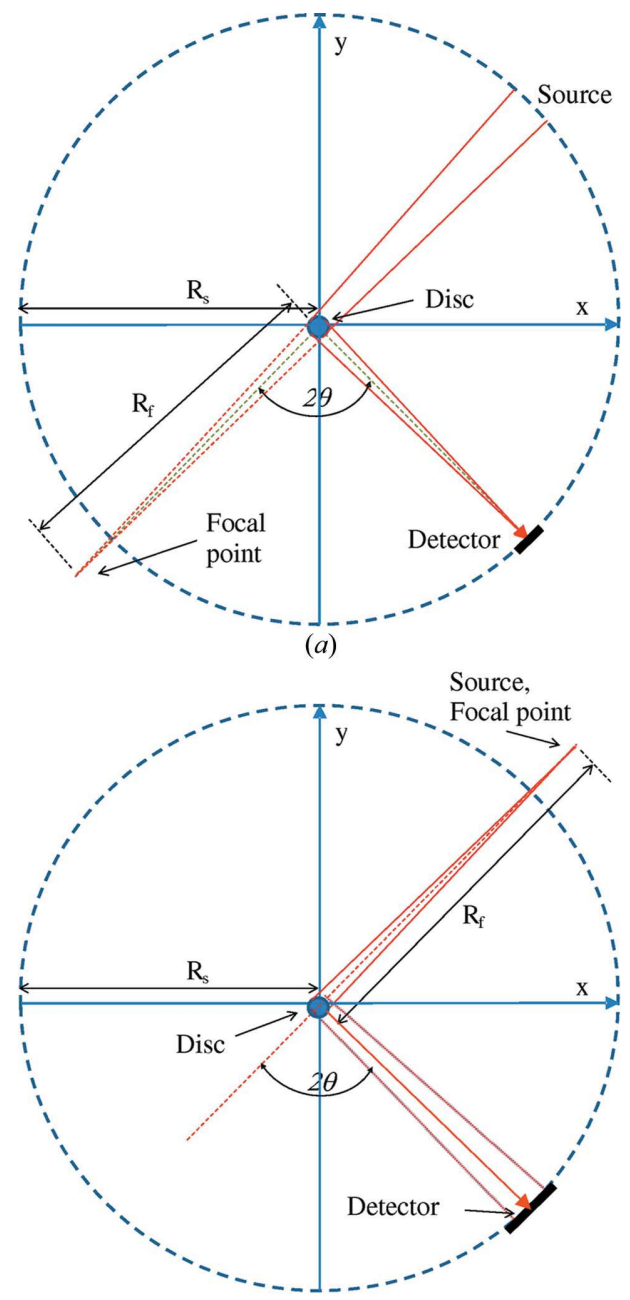

(b)

Figure 1

(a) Convergent and (b) divergent beam geometries. $R_{\mathrm{s}}=$ diffractometer radius, $R_{\mathrm{f}}=$ focal length. With the origin at the centre of the disc, the detector moves along the diffractometer circle from $\left(R_{\mathrm{s}}, 0\right)$ to $\left(0,-R_{\mathrm{s}}\right)$ and the source moves from $\left(0, R_{\mathrm{s}}\right)$ to $\left(R_{\mathrm{s}}, 0\right)$. Both the source and the detector move such that a source position $(x, y)$ corresponds to a detector position $(x,-y)$. both cases, the measured $2 \theta$ positions are determined with the focal point considered as the source of the rays. All analyses are performed using the TOPAS-Academic software (Coelho, 2017).

We first consider the aberration arising from a line on the disc parallel to the $x$ axis for $x>0$, which we will call line-ondisc (see Fig. 2). The intensity contribution from the line is given by equation (1), where $r$ is the radius of the disc, $\mu$ is the linear absorption coefficient of the material taking into account its packing density and $P(x, y, 2 \theta)$ is the path length through the disc at $x$ for a Bragg angle of $2 \theta$ :

$$
I(y, 2 \theta)=\int_{0}^{r_{y}} \exp [-\mu P(x, y, 2 \theta)] \mathrm{d} x, \text { where } \quad r_{y}=\left(r^{2}-y^{2}\right)^{1 / 2} \text {. }
$$

The intensity contribution from the line does not determine where on the $2 \theta$ axis the intensity should be placed in order to form the aberration. The line is therefore broken into segments and the intensity contribution from each segment transformed on to the corresponding $2 \theta$ axis of the diffraction pattern. Too few segments along the line will produce inaccurate aberration shapes and intensities. The integral of equation (1) for $I(x, y)$ can be efficiently evaluated numerically by choosing values of $x$ that vary as a function of $\mu$ such that the variation of $P(x, y, 2 \theta)$ within a particular segment can be considered linear. The resulting integral at one of the discrete points, $x_{j}$, at the midpoint of a segment is

$$
\begin{aligned}
I\left(x_{j}, y, 2 \theta\right) & =\int_{x_{1, j}}^{x_{2, j}} \exp \left[-\mu\left(m_{j} x+c_{j}\right)\right] \mathrm{d} x, \\
\text { where } \quad x_{1, j} & =\frac{1}{2}\left(x_{j-1}+x_{j}\right), \quad x_{2, j}=\frac{1}{2}\left(x_{j+1}+x_{j}\right), \\
m_{j} & =\frac{P\left(x_{2, j}, y, 2 \theta\right)-P\left(x_{1, j}, y, 2 \theta\right)}{x_{2, j}-x_{1, j}}, \\
c_{j} & =P\left(x_{2, j}, y, 2 \theta\right)-m_{j} x_{2, j} .
\end{aligned}
$$

For accuracy, $I\left(x_{j}, y, 2 \theta\right)$ for each $x_{j}$ should be equal, or $I\left(x_{j+1}, y, 2 \theta\right)=I\left(x_{j}, y, 2 \theta\right)$. This condition can be met by setting $P\left(x_{j+1}, y, 2 \theta\right)-P\left(x_{j}, y, 2 \theta\right)=c$, where $c$ is some chosen change in path length. For $2 \theta=180^{\circ}$ and for $y=0$, this condition can be met exactly by solving for $x_{j}$ in equation (3):

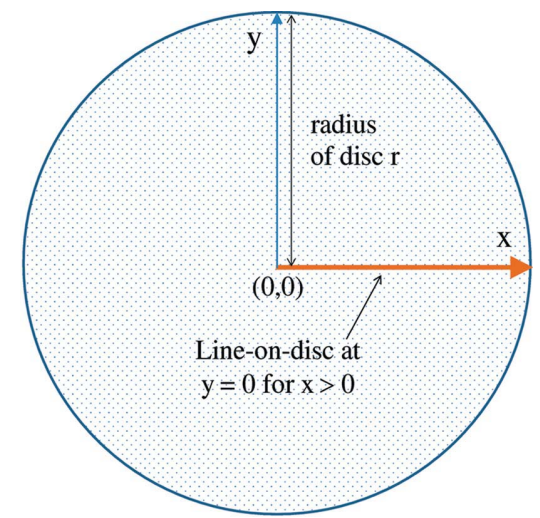

Figure 2

Schematic of the disc and the line-on-disc with the origin at the centre of the disc. 


$$
\begin{aligned}
& I\left(x_{j}, 0,90\right)=\int_{x_{1, j}}^{x_{2, j}} \exp \left[-\mu\left(r_{y}-x\right) \mathrm{d} x=\Delta A,\right. \\
& \text { where } \Delta A
\end{aligned}
$$

Or, $x_{j}=r_{y}+\ln (1-j \mu \Delta A) / \mu$, where $N_{y}$ corresponds to the number of chosen $x_{j}$ points and $A$ is the total intensity along the line-on-disc at $y=0$. For $2 \theta<180^{\circ}$, equation (2) needs to be equated to $\triangle A$ and then solved numerically for $x_{j}$. The positions of $x_{j}$ for various $2 \theta$ are shown by the markers (circles) in Fig. 3. These path-length curves are independent of $\mu$; however, the positions of the $x_{j}$ points are dependent on $\mu$. As can be seen, a linear approximation of path length becomes less accurate as $2 \theta$ decreases, leading to inaccurate $I\left(x_{j}, 0,2 \theta\right)$ values. Solving equation (2) for $x_{j}$ numerically for $2 \theta<180^{\circ}$ is computationally expensive and serves only to place $x_{j}$ such that $I\left(x_{j+1}, 0,2 \theta\right)=I\left(x_{j}, 0,2 \theta\right)$ for all $j$. To approximately do the same, equation (3) can be used for all $2 \theta$ with additional points added for low $2 \theta$. These additional points can be added by compressing the $x$ axis; preliminary analysis has shown that multiplying the $x$ axis by $q=\frac{1}{2}[1+\sin (\theta)]$ works well, as described in equation (4):

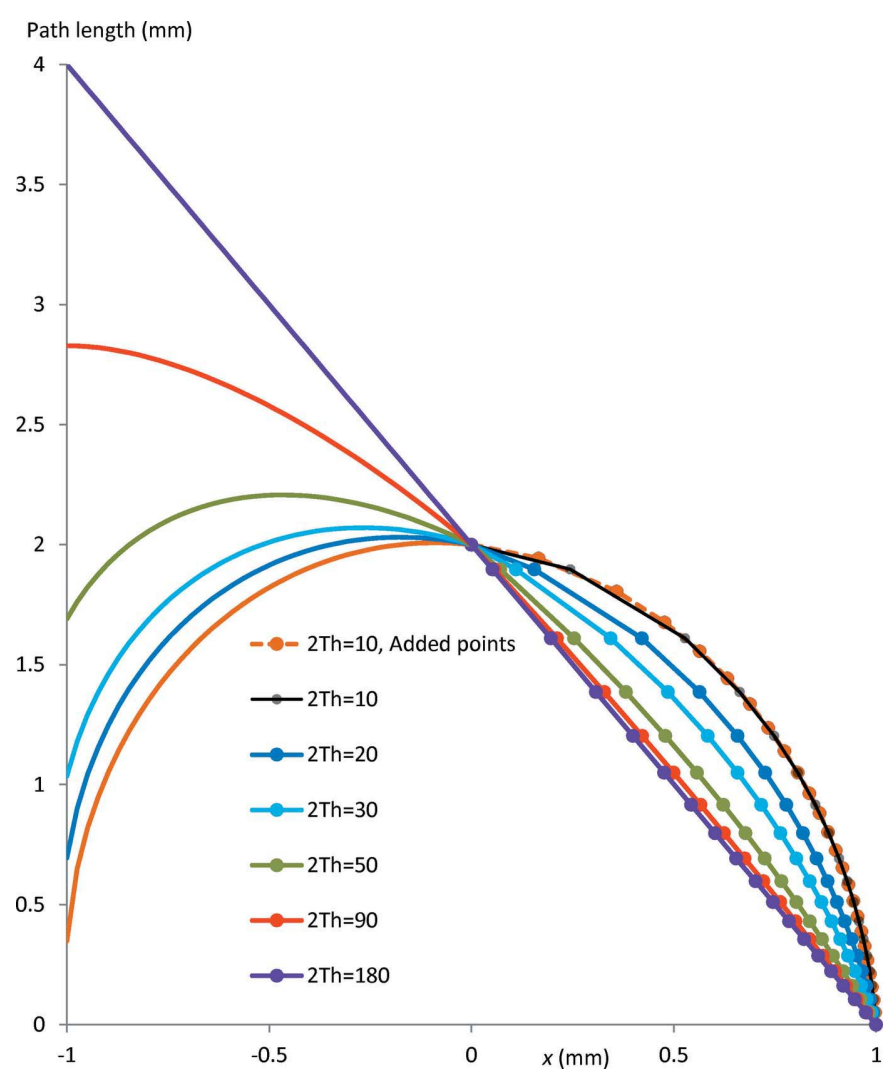

Figure 3

Path length through the disc as a function of $x$ at $y=0$ for various $2 \theta$ values. For the solid lines, the positions of the $x_{j}$ values (the markers) were determined using equation (4) for $\mu=20 \mathrm{~cm}^{-1}$. For the dashed line, corresponding to $10^{\circ} 2 \theta$, the $x_{j}$ values were determined using equation (5).

$$
\begin{aligned}
& \int_{x_{1, j}}^{x_{2, j}} \exp \left[-\mu\left(r_{y}-q x\right) \mathrm{d} x=\Delta A\right. \\
& \text { or } \quad x_{j}=r_{y}+\ln (1-j \mu q \Delta A) /(\mu q) .
\end{aligned}
$$

Additionally, for $y \neq 0$, the path length versus $x$ curves, similar to Fig. 3, have an excess of points with increasing $y$. In this case, some $x_{j}$ points are removed by expanding the $x$ axis by dividing the step size in $x_{j}$ by $r_{y} / r$, resulting in the final $x_{j}$ determination equation of

$$
x_{j}=r_{y}+\ln (1-j \mu q \Delta A) r /\left(\mu q r_{y}\right) \text {. }
$$

Equation (5) concentrates the $x_{j}$ points towards the edge of the disc for high-absorbing materials and more evenly across the disc for low-absorbing materials; this is shown for various $2 \theta$ and $\mu$ in Fig. 4. Also shown is the increase in the number of points for low $2 \theta$ values as $\mu$ increases.

At small $2 \theta$ angles, rays passing close to the left edge of the disc can contribute in a nontrivial manner. Thus, $x_{j}$ values should also occur more frequently as $x$ approaches $-r_{y}$. This is achieved by setting the $x_{j}$ values for $x_{j}<0$ to the negative of the $x_{j}$ values for $x_{j}>0$. Thus, stepping along a line parallel to the $x$ axis is performed as follows:

(1) Calculate $I\left(x_{j}, y\right)$ for $x_{j}>0$ starting with $x_{2, j}$ at $r_{y}$.

(2) Calculate $I\left(-x_{j}, y\right)$ for $x_{j}<0$ starting with $x_{1, j}$ at $-r_{y}$.

For case (1) the path length through the specimen for a ray diffracting at $x$, for $x>0$, is greater than that for $x+\Delta$, where $\Delta$ is some positive value, that is, $I\left(x_{j}, y\right)$ is always less than $I\left(x_{j}+\Delta, y\right)$. Thus, calculation of $I\left(x_{j}, y\right)$ can be terminated after the intensity drops off to a sufficiently small value, $t=10^{-4}$, or calculation is terminated when $\exp \left[-\mu P\left(x_{j}, 2 \theta\right)\right]<t$. For case (2) and starting at $x=-r$ the path length is more complicated as $x$ increases; it is also a function of $2 \theta$, as seen in Fig. 3. However, the path length is always greater at either $x=-r$ or $x=0$. Termination of the calculation can therefore occur when both $\exp \left[-\mu P\left(x_{j}, 2 \theta\right)\right]<$ $t$ and $\exp \left[-\mu P\left(x_{j}=0,2 \theta\right)\right]<t$.

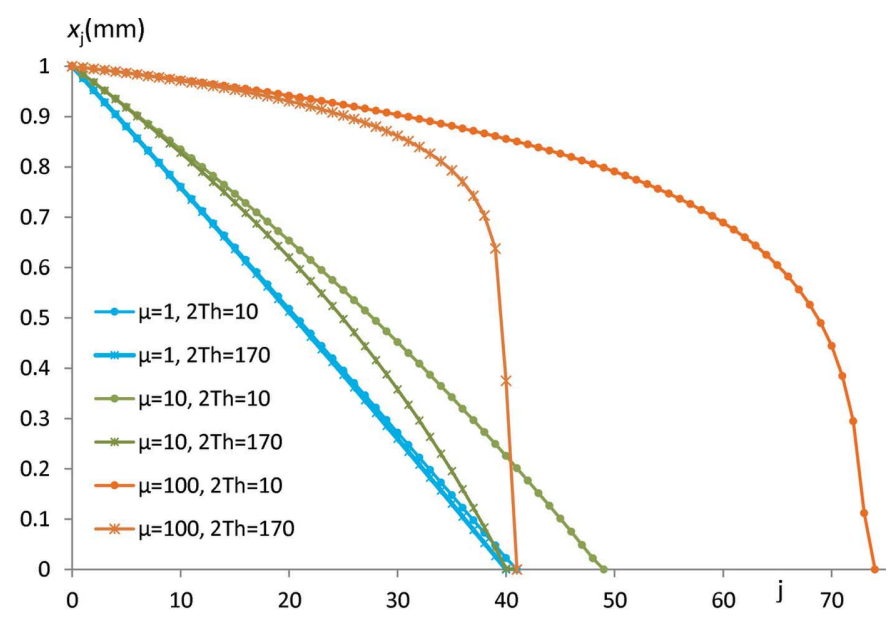

Figure 4

$x_{j}$ determined using equation (5) versus $j$ for various $2 \theta$ and $\mu$ at $y=0$ for $N_{y}=40$ and $r=1 \mathrm{~mm}$. $\mu$ values in $\mathrm{cm}^{-1}$ and $2 \theta$ values in ${ }^{\circ}$. 
Fig. 5 shows a representation of $I(x, y)$ for all $x$ and $y$ within the disc for the cases of $N_{y}=100$ and 40; the $x_{j}$ positions can be seen as smeared dots. The smaller $N_{y}=40$ case is computationally fast and produces accurate aberrations with regard to intensities, shapes and positions.

\section{Creating a $2 \theta$ scan from the diffracting intensities on the disc}

A diffraction pattern, $L(2 \varphi)$, in the present context, comprises line segments connecting equally spaced points. The intensity contribution, $I\left(x_{j}, y\right)$, at the point $\left(x_{j}, y\right)$ on the disc is detected at $2 \varphi_{\mathrm{d}}\left(x_{j}, y\right)$, where $2 \varphi_{\mathrm{d}}\left(x_{j}, y\right)$ is also a function of the focal length, $R_{\mathrm{f}}$, and the specimen-to-detector distance, $R_{\mathrm{s}} . I\left(x_{j}, y\right)$ is transformed to $L(2 \varphi)$ by splitting $I\left(x_{j}, y\right)$ into two $2 \varphi$ points, $2 \varphi_{1}$ and $2 \varphi_{2}$, corresponding to $2 \varphi$ just before and just after $2 \varphi_{\mathrm{d}}$, respectively, as described by equation (6):

$$
\begin{aligned}
& I\left(x_{j}, y\right)\left(2 \varphi_{2}-2 \varphi_{\mathrm{d}}\right) / \Delta 2 \varphi \quad \text { added to } L\left(2 \varphi_{1}\right), \\
& I\left(x_{j}, y\right)\left(2 \varphi_{\mathrm{d}}-2 \varphi_{1}\right) / \Delta 2 \varphi \quad \text { added to } L\left(2 \varphi_{2}\right), \\
& \text { where } \Delta 2 \varphi=\left(2 \varphi_{2}-2 \varphi_{1}\right) .
\end{aligned}
$$

The two points, $L\left(2 \varphi_{1}\right)$ and $L\left(2 \varphi_{2}\right)$, of equation (6) have the same moment as $L\left(2 \varphi_{\mathrm{d}}\right)$ and the same intensity as the original intensity, or $L\left(2 \varphi_{\mathrm{d}}\right)=L\left(2 \varphi_{1}\right)+L\left(2 \varphi_{2}\right)$. The intensity and centroid of the aberration is therefore independent of the $2 \varphi$ axis position. For a single point, the integral breadth of equation (6) is equivalent to $\Delta 2 \varphi$. This introduces a small amount of broadening in the final profile, which, in practice, is negligible and can only be observed, in a least-squares sense, if the observed profile is re-binned numerically at smaller step sizes than the original step size [equation (8), Coelho et al. (2015)]. For example, consider a line profile, $L$, comprising a single nonzero point. Fitting a delta function convoluted with two impulse functions to $L$, using TOPAS-Academic, results in the width of the impulse function refining to zero. Re-binning $L$ at much smaller steps, $\Delta 2 \varphi_{\text {small }}$, and again fitting a delta function with two impulse functions, results in the width of both impulse functions refining to a value equal to the original $\Delta 2 \varphi$. Detection of the extra broadening introduced by equation (6) is therefore not typically observed, as scan data are

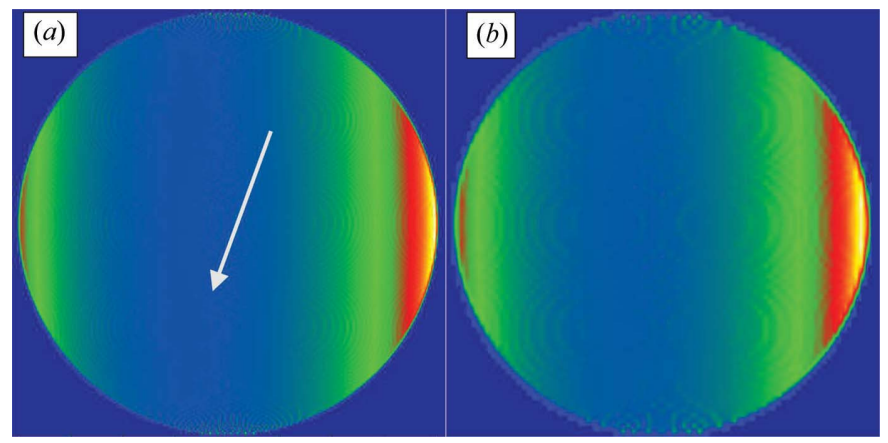

Figure 5

$I(x, y)$ for $\mu=15 \mathrm{~cm}^{-1}$ and for $20^{\circ} 2 \theta$ for $(a) N_{y}=100$ and $(b) N_{y}=40$. Red and yellow show areas of high intensity. The coordinate system is as outlined in Fig. 2. The arrow indicates the direction of the incident beam. often re-binned at step sizes larger than the original step size and not at smaller step sizes.

Fig. 6 shows the aberration produced using equation (6) for a line through the disc at $y=0$ for the defocused case of $R_{\mathrm{f}}>R_{\mathrm{s}}$ and for two $\Delta 2 \varphi$ step sizes. The ripples at small $\Delta 2 \varphi$ are due to the discrete nature of the process. Nevertheless, the aberration has the correct intensity and centroid moment. The ripples for $\Delta 2 \varphi=0.001^{\circ} 2 \theta$ can be removed by convoluting three impulse functions of width $H$, as defined in equation (7), where $2 \varphi\left(x_{j}, y\right)$ is the detected $2 \theta$ position of the ray diffracting from the $\left(x_{j}, y\right)$ position on the disc:

$$
H=\max \left[\left|2 \varphi\left(x_{j+1}, y\right)-2 \varphi\left(x_{j}, y\right)\right|\right] \text { for all } j .
$$

The impulse functions are symmetric and normalized and hence do not change the intensity or centroid of $L(2 \varphi)$. Fig. 7 shows aberrations from a line through the disc after applying the three smoothing impulse function convolutions with width $H$ for various $N_{y}$ and $y$ values for $\Delta 2 \varphi=0.001^{\circ} 2 \varphi$; as can be

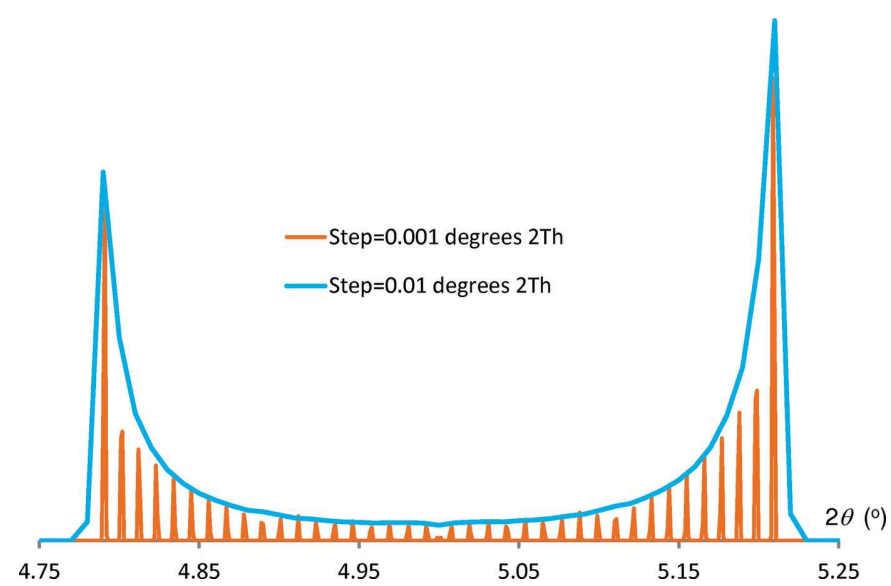

Figure 6

Convergent beam geometry aberration for a line-on-disc lying on the $x$ axis at $y=0$ for $N_{y}=20$, with $\Delta 2 \varphi=0.01$ and $0.001^{\circ} 2 \theta . r=1 \mathrm{~mm}, R_{\mathrm{s}}=$ $200 \mathrm{~mm}, R_{\mathrm{f}}=800 \mathrm{~mm}$ and $\mu=20 \mathrm{~cm}^{-1}$.

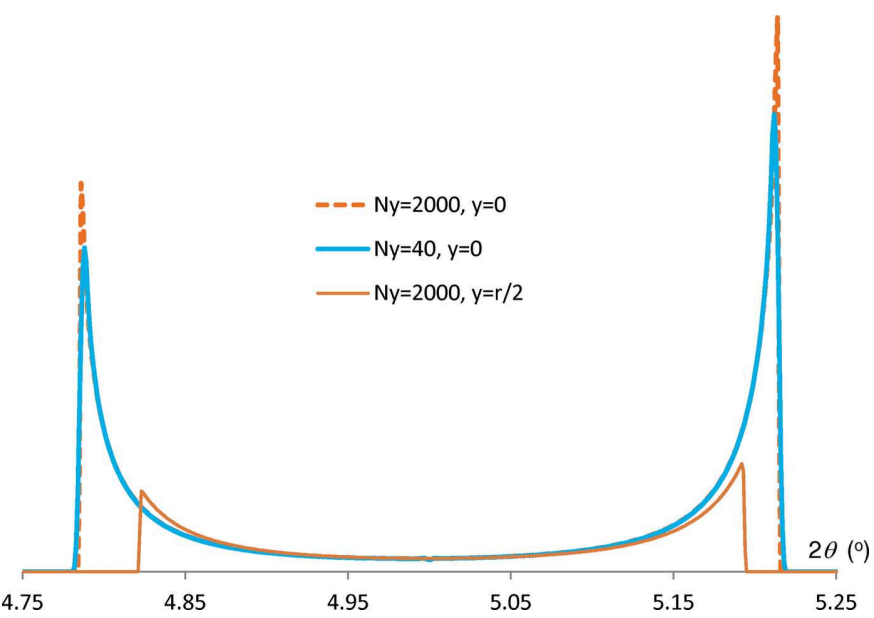

Figure 7

Convergent beam geometry aberrations for a line-on-disc parallel to the $x$ axis for various $N_{y}$ and $y$ values for $\Delta 2 \varphi=0.001^{\circ} 2 \theta . r=1 \mathrm{~mm}, R_{\mathrm{s}}=$ $200 \mathrm{~mm}, R_{\mathrm{f}}=800 \mathrm{~mm}$ and $\mu=20 \mathrm{~cm}^{-1}$. 
seen, the ripples have been smoothed and the aberration looks very similar to the aberration for $N_{y}=2000$; the latter has a much smaller $H$ applied.

\section{Summing line-on-disc aberrations to form the final disc aberration}

Summing $N_{y}$ line aberrations with $y$ values equally spaced from $-r$ to $r$ produces the final aberration, but with bumps corresponding to the use of discrete $y$ values. The final aberration can be smoothed by including the difference in $2 \varphi$ between the line-on-disc aberrations. The enhanced $H$ definition is given by

$$
\begin{aligned}
H=\max & {\left[\left|2 \varphi\left(x_{j+1}, y\right)-2 \varphi\left(x_{j}, y\right)\right|,\right.} \\
& \left.\left|2 \varphi\left(x=0, y_{k+1}\right)-2 \varphi\left(x=0, y_{k}\right)\right|\right] \text { for all } j .
\end{aligned}
$$

Fig. 8 shows the final aberration for a convergent and divergent beam with and without the application of the three impulse smoothing convolutions. As can be seen, the application of the convolutions for the $N_{y}=40$ case produces an

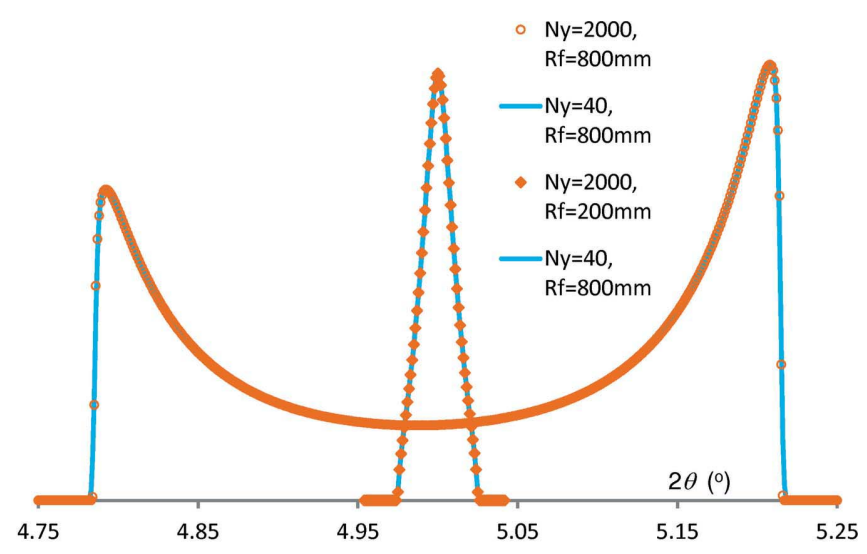

(a)

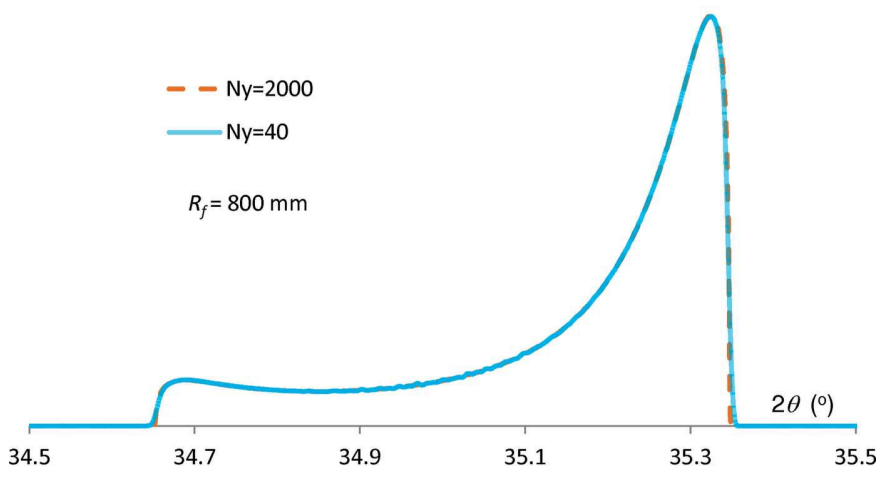

Figure $8 \quad(b)$

(a) Convergent and (b) divergent beam geometry disc aberrations for various $N_{y}$ and $R_{\mathrm{f}}$ obtained by summing line aberrations. $r=1 \mathrm{~mm}, R_{\mathrm{s}}=$ $200 \mathrm{~mm}$ and $\mu=20 \mathrm{~cm}^{-1}$. For $N_{y}=40$, the smoothing impulse convolutions defined in equation (8) have been applied. They were not applied for $N_{y}=2000$. almost identical aberration to the $N_{y}=2000$ case where the impulse convolutions were not applied.

For accurate integrated intensities at very low $\mu, \mu<$ $0.1 \mathrm{~cm}^{-1}$ depending on $r$, only two $x_{j}$ points are needed per $y$ line-on-disc $\left(x_{1, j}=0\right.$ and $\left.x_{2, j}=r_{y}\right)$. However, such a low number of points would not accurately describe the shape of the aberration in $2 \theta$ space; hence the use of equation (5) in determining $x_{., j}$ values.

\section{Comparison with ray tracing}

The present disc aberration was compared with a ray-tracing approach (Appendix $A$ ) over the $2 \theta$ range of 5 to $175^{\circ}$ for $(a)$ convergent $\left(R_{\mathrm{f}}=200 \mathrm{~mm}\right),(b)$ semi-convergent $\left(R_{\mathrm{f}}=\right.$ $800 \mathrm{~mm}),(c)$ divergent $\left(R_{\mathrm{f}}=200 \mathrm{~mm}\right)$ and $(d)$ parallel incident beams for $R_{\mathrm{s}}=200 \mathrm{~mm}, r=1 \mathrm{~mm}$ and $\mu=20 \mathrm{~cm}^{-1}$. The peak intensities, shapes and positions all closely agreed (see Fig. 14 in Appendix $A$ ).

\section{Speed and approximating disc aberrations from nearby disc aberrations}

For $N_{y}=40$, present laptop computers can calculate $\sim 20000$ disc aberrations per second; however, using the aberration buffer of TOPAS-Academic, no more than $\sim 30$ disc aberrations need be calculated for a particular geometry. The change in disc aberration shapes as a function of $2 \theta$, as seen in Fig. 9, is smooth and can be considered linear over a small $2 \theta$ range. Aberrations at a particular $2 \theta$ can therefore be approximated from nearby calculated aberrations; this approximation is very accurate when the nearby calculated aberrations are no more than $2^{\circ} 2 \theta$ apart, or the distance between calculated aberrations is limited to $4^{\circ} 2 \theta$. Equation (9) shows the formulation of the approximation:

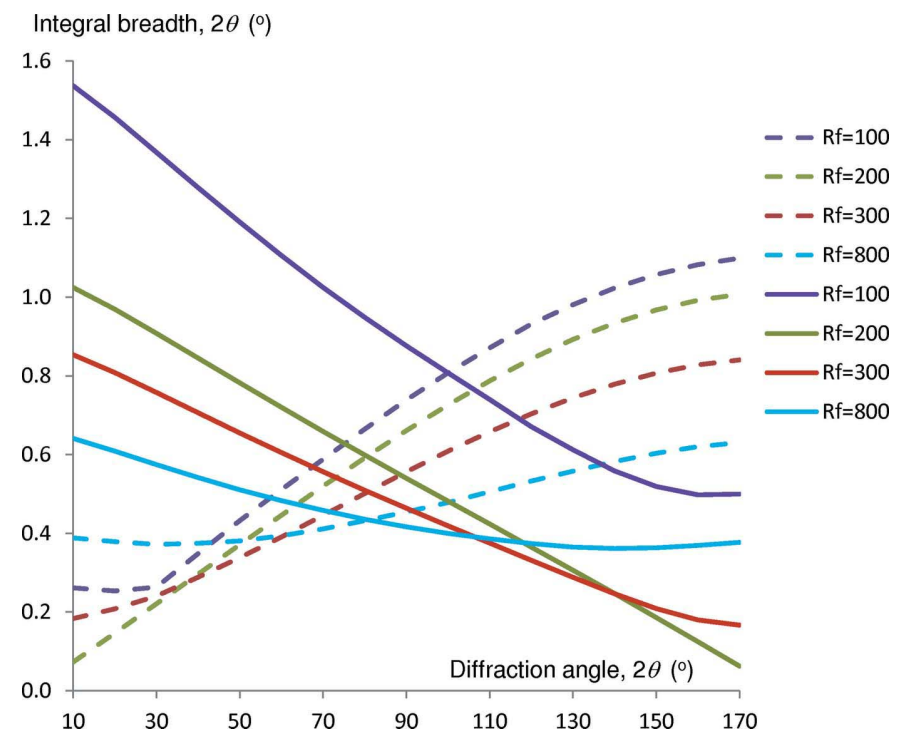

Figure 9

Integral breadths for convergent (dashed lines) and divergent (solid lines) beam geometries for disc aberrations as a function of $2 \theta$ and $R_{\mathrm{f}}$. $R_{\mathrm{f}}$ values in mm. $r=1 \mathrm{~mm}, R_{\mathrm{s}}=200 \mathrm{~mm}$ and $\mu=5 \mathrm{~cm}^{-1}$. 
Table 1

(a) Convergent and (b) divergent beam geometry disc aberration statistics for $r=1 \mathrm{~mm}, R_{\mathrm{s}}=200$ and $\Delta 2 \varphi=0.001^{\circ} 2 \theta$ for 17 peaks in the range $10-170^{\circ} 2 \theta$ for various focal lengths, $R_{\mathrm{f}}$, and absorption coefficients, $\mu$.

$\Delta$ Centroid and $\Delta I$ correspond to the centroid difference in degrees and the percentage difference in integrated intensity, respectively, between aberrations calculated with $N_{y}=40$ and $N_{y}=2000$. Min: minimum; Max: maximum; Stdev: standard deviation.

(a) Convergent beam geometry.

$$
\mu\left(\mathrm{cm}^{-1}\right)
$$

$R_{\mathrm{f}}$ $(\mathrm{mm})$ 5 10 20 50 100

100 $\begin{array}{lrrrrr}\text { Min Centroid } & -0.036462 & -0.074507 & -0.143973 & -0.247643 & -0.273500 \\ \text { Max Centroid } & -0.005513 & -0.009507 & -0.013640 & -0.016972 & -0.018181 \\ \text { Min } \Delta \text { Centroid } & 0.000002 & 0.000006 & 0.000007 & -0.000018 & -0.000026\end{array}$ $\begin{array}{llllll}\text { Max } \Delta \text { Centroid } & 0.000011 & 0.000031 & 0.000072 & 0.000145 & 0.000055\end{array}$ $\begin{array}{llllll}\text { Stdev } \Delta \text { Centroid } & 0.000003 & 0.000008 & 0.000022 & 0.000044 & 0.000023\end{array}$

200 Min Centroid Max Centroid Min $\Delta$ Centroid Max $\Delta$ Centroid Stdev $\Delta$ Centroid

$300 \quad$ Min Centroid Max Centroid Min $\triangle$ Centro Max $\Delta$ Centroid Stdev $\Delta$ Centroid

0.000065 0.000401 0.000000 0.000000

0.000076 0.000100 $0.000484 \quad 0.000693$ $0.000000 \quad 0.000000$

0.000133 0.001035

0.000149 $\begin{array}{ccc}0.000000 & 0.000000\end{array}$ $\begin{array}{lll}0.000000 & 0.000000 & 0.000000\end{array}$ 0.000000 $\begin{array}{lllll}0.001923 & 0.003271 & 0.004684 & 0.005846\end{array}$ $\begin{array}{rrrr}0.012694 & 0.025486 & 0.048916 & 0.083482\end{array}$ $-0.000004-0.000010-0.000024-0.000049$

0.000015

Min Centroid Max Centroid 0.004245 Min $\Delta$ Centroid Max $\Delta$ Centroid $\begin{array}{llll}0.028070 & 0.056785 & 0.109336 & 0.187165\end{array}$

$-0.000009-0.000023-0.000055-0.000109-($ $-0.000002-0.000005-0.000005$ Stdev $\Delta$ Centroid

$0.000002-0.000006-0.000017$

$0.000006 \quad 0.000017$

0.001163

0.000000

0.000000 0.000000

0.006274 0.091891 $-0.000019$ 0.000009 0.000008 $\begin{array}{llll}-0.000001 & -0.000002 & -0.000002 & 0.000007\end{array}$

Convergent and divergent beam geometry intensity statistics for all $R_{\mathrm{f}}$

$\begin{array}{llrrrr}\text { Min \% } \Delta I & 0.022 & -0.015 & -0.115 & -0.555 & -1.136 \\ \text { Max \% } \Delta I & 0.089 & 0.116 & 0.149 & 0.202 & 0.191 \\ \text { Stdev \% } \Delta I & 0.021 & 0.040 & 0.076 & 0.182 & 0.314\end{array}$

(b) Divergent beam geometry.

\begin{tabular}{|c|c|c|c|c|c|c|}
\hline \multirow[b]{2}{*}{$\begin{array}{l}R_{\mathrm{f}} \\
(\mathrm{mm})\end{array}$} & & \multicolumn{5}{|l|}{$\mu\left(\mathrm{cm}^{-1}\right)$} \\
\hline & & 5 & 10 & 20 & 50 & 100 \\
\hline \multirow[t]{5}{*}{100} & Min Centroid & 0.016763 & 0.028827 & 0.041434 & 0.051757 & 0.055547 \\
\hline & Max Centroid & 0.111218 & 0.226219 & 0.436775 & 0.748723 & 0.825092 \\
\hline & Min $\Delta$ Centroid & -0.000036 & -0.000095 & -0.000220 & -0.000438 & -0.000166 \\
\hline & Max $\Delta$ Centroid & -0.000008 & -0.000019 & -0.000021 & 0.000060 & 0.000084 \\
\hline & Stdev $\Delta$ Centroid & 0.000009 & 0.000024 & 0.000067 & 0.000135 & 0.000072 \\
\hline \multirow[t]{5}{*}{200} & Min Centroid & 0.011203 & 0.019243 & 0.027637 & 0.034498 & 0.037013 \\
\hline & Max Centroid & 0.074238 & 0.150827 & 0.291003 & 0.498796 & 0.549773 \\
\hline & Min $\Delta$ Centroid & -0.000024 & -0.000063 & -0.000147 & -0.000292 & -0.000111 \\
\hline & Max $\Delta$ Centroid & -0.000005 & -0.000013 & -0.000014 & 0.000039 & 0.000055 \\
\hline & Stdev $\Delta$ Centroid & 0.000006 & 0.000016 & 0.000045 & 0.000090 & 0.000048 \\
\hline \multirow[t]{5}{*}{300} & Min Cen & 0.009348 & 0.016049 & 0.023042 & 0.028756 & 0.03085 \\
\hline & Max Centroid & 0.061920 & 0.125727 & 0.242499 & 0.415610 & 0.458098 \\
\hline & Min $\Delta$ Centroid & -0.000020 & -0.000052 & -0.000122 & -0.000243 & -0.000092 \\
\hline & Max $\Delta$ Centroid & -0.000004 & -0.000011 & -0.000011 & 0.000033 & 0.000046 \\
\hline & Stdev $\Delta$ Centroid & 0.000005 & 0.000013 & 0.000037 & 0.000075 & 0.000040 \\
\hline \multirow[t]{5}{*}{800} & Min Centr & 0.007029 & 0.012056 & 0.017301 & 0.021587 & 0.023158 \\
\hline & Max Centroid & 0.046529 & 0.094375 & 0.181930 & 0.311713 & 0.343572 \\
\hline & entroid & -0.000015 & -0.000039 & -0.000092 & -0.000182 & -0.000069 \\
\hline & Max $\Delta$ Centroid & -0.000003 & -0.000008 & -0.000008 & 0.000024 & 0.000034 \\
\hline & Stdev $\Delta$ Centroid & 0.000004 & 0.000010 & 0.000028 & 0.000056 & 0.000030 \\
\hline
\end{tabular}

$$
\begin{aligned}
A_{2}\left(\varepsilon_{2}\right) & =A_{1}\left(\varepsilon_{1}\right) \alpha+A_{3}\left(\varepsilon_{3}\right)(1-\alpha), \\
\text { where } \quad \varepsilon_{n} & =2 \varphi-2 \theta_{n} \\
\text { and } \quad \alpha & =\left(2 \theta_{3}-2 \theta_{2}\right) /\left(2 \theta_{3}-2 \theta_{1}\right) .
\end{aligned}
$$

Fig. 10 shows the very good approximation for $2 \theta=7^{\circ}$ using aberrations calculated at 5 and $9^{\circ}$; this is an extreme case where $R_{\mathrm{f}} \gg R_{\mathrm{s}}$ and $2 \theta_{1}$ is at the low angle of $5^{\circ}$. The use of the approximation of equation (9) results in $\sim 30$ aberration calculations, even when hundreds of diffraction patterns each with thousands of peaks are synthesized, and is an important factor in speeding up large Rietveld refinements (Rietveld, 1969).

\section{Preliminary analysis}

Table 1 shows statistics obtained for convergent and divergent beam geometries for various $R_{\mathrm{f}}$ and $\mu$ for $N_{y}=$ 40 compared to the same aberrations produced with $N_{y}=$ 2000 . These data represent a broad range of $\mu r(0-10)$ and $r / R_{\mathrm{f}}$ values, demonstrating the accurate description of the present disc aberration. Aberrations with $\mu r>10$ are also accurate because of the variable step size as determined by equation (5). Table 2 shows the small integral breadth variations between $N_{y}=40$ and $N_{y}=2000$; for accuracy, the aberrations for integral breadth calculations were carried out with a very small step size of $\Delta 2 \varphi=0.0001^{\circ} 2 \theta$. The case of $R_{\mathrm{s}}=R_{\mathrm{f}}$ corresponds to the worst-case scenario, as the focusing condition is maximized and the peaks are therefore at their sharpest at low angles. The case in Table 2 with a percentage difference greater than $1 \%$ occurs for the high-absorbing $\mu=100 \mathrm{~cm}^{-1}$; at this high $\mu$, the small diffraction volume results in a small integral breadth of $0.0133^{\circ} 2 \theta$. Fig. 9 shows the increase in broadening for both convergent and divergent beam geometries as $\left|R_{\mathrm{f}}-R_{\mathrm{s}}\right|$ increases; for convergent beam geometry, broadening increases with increasing $2 \theta$, whereas for divergent beam geometry, broadening decreases with increasing $2 \theta$. These trends can be understood by considering the case $\mu \rightarrow 0$, where from symmetry and the position of the focal point a disc aberration for convergent beam geometry at $2 \theta$ is the same as a disc aberration for divergent beam geometry at $180-2 \theta$. Note that $x_{j}$ points for low $\mu$ are similar for both high and low $2 \theta$ angles, as shown in Fig. 4.

Fig. 11 shows an $I(x, y)$ representation of the highabsorbing material $\mathrm{LaB}_{6}$, where $\mu$ is set to the expected value for molybdenum $K \alpha$ radiation. The small diffraction region with appreciable intensity for the low angle of $20^{\circ} 2 \theta$, relative to the larger diffraction volume at $120^{\circ} 2 \theta$, is clear.

\section{Analysis of $\mathrm{LaB}_{6}$ data}

$\mathrm{LaB}_{6}$ powder, NIST SRM 660a (NIST, 2000), with a certified lattice parameter of 0.41569162 (97) $\mathrm{nm}$ at 295.7 K, was mounted in a capillary with an estimated 
Table 2

Convergent beam geometry integral breadth (IB) calculations for $\Delta 2 \varphi=$ $0.0001^{\circ} 2 \theta$ with $R_{\mathrm{s}}=R_{\mathrm{f}}=200 \mathrm{~mm}$ and $r=1 \mathrm{~mm}$.

IB corresponds to the value obtained with $N_{y}=2000 ; \Delta \mathrm{IB}$ corresponds to the difference between integral breaths calculated at $N_{y}=2000$ and $N_{y}=40$.

\begin{tabular}{|c|c|c|c|c|c|c|}
\hline \multirow[b]{2}{*}{$\begin{array}{l}\text { Bragg angle } \\
\left({ }^{\circ} 2 \theta\right)\end{array}$} & \multicolumn{3}{|c|}{$\mu=5 \mathrm{~cm}^{-1}$} & \multicolumn{3}{|c|}{$\mu=100 \mathrm{~cm}^{-1}$} \\
\hline & $\begin{array}{l}\text { IB } \\
\left({ }^{\circ} 2 \theta\right)\end{array}$ & $\begin{array}{l}\Delta \mathrm{IB} \\
\left({ }^{\circ} 2 \theta\right)\end{array}$ & $\begin{array}{l}\Delta \mathrm{IB} \\
(\%)\end{array}$ & $\begin{array}{l}\text { IB } \\
\left({ }^{\circ} 2 \theta\right)\end{array}$ & $\begin{array}{l}\Delta \mathrm{IB} \\
\left({ }^{\circ} 2 \theta\right)\end{array}$ & $\begin{array}{l}\Delta \mathrm{IB} \\
(\%)\end{array}$ \\
\hline 10 & 0.0728 & 0.0001 & 0.1175 & 0.0133 & 0.0004 & 3.1854 \\
\hline 20 & 0.1467 & -0.0002 & -0.1098 & 0.0376 & 0.0003 & 0.7055 \\
\hline 30 & 0.2209 & 0.0000 & 0.0028 & 0.0721 & 0.0005 & 0.6241 \\
\hline 40 & 0.2960 & -0.0001 & -0.0288 & 0.1173 & 0.0004 & 0.3171 \\
\hline 50 & 3710 & 0.0000 & -0.0117 & 0.1721 & 0.0004 & 0.2225 \\
\hline 60 & 0.4458 & 0.0000 & -0.0026 & 0.2359 & 0.0004 & 0.1692 \\
\hline 70 & .5196 & 0.0000 & -0.0078 & 0.3078 & 0.0003 & 0.1063 \\
\hline 80 & 0.5915 & -0.0001 & -0.0181 & 0.3866 & 0.0003 & 0.0885 \\
\hline 90 & 6609 & -0.0002 & -0.0276 & 0.4713 & 0.0003 & 0.0709 \\
\hline 100 & 7265 & -0.0002 & -0.0216 & 0.5601 & 0.0005 & 0.0956 \\
\hline 110 & 0.7878 & -0.0004 & -0.0469 & 0.6519 & 0.0004 & 0.0676 \\
\hline 120 & 0.8436 & -0.0004 & -0.0470 & 0.7444 & 0.0003 & 0.0422 \\
\hline 130 & 0.8929 & -0.0005 & -0.0608 & 0.8356 & 0.0005 & 0.0605 \\
\hline 140 & 0.9351 & -0.0009 & -0.0989 & 0.9229 & 0.0003 & 0.0295 \\
\hline 150 & 9689 & -0.0012 & -0.1224 & 1.0026 & -0.0008 & -0.0751 \\
\hline 160 & 9937 & -0.0016 & -0.1575 & 1.0705 & -0.0015 & -0.1412 \\
\hline 170 & 1.0089 & -0.0018 & -0.1822 & 1.1199 & -0.0043 & -0.3857 \\
\hline
\end{tabular}

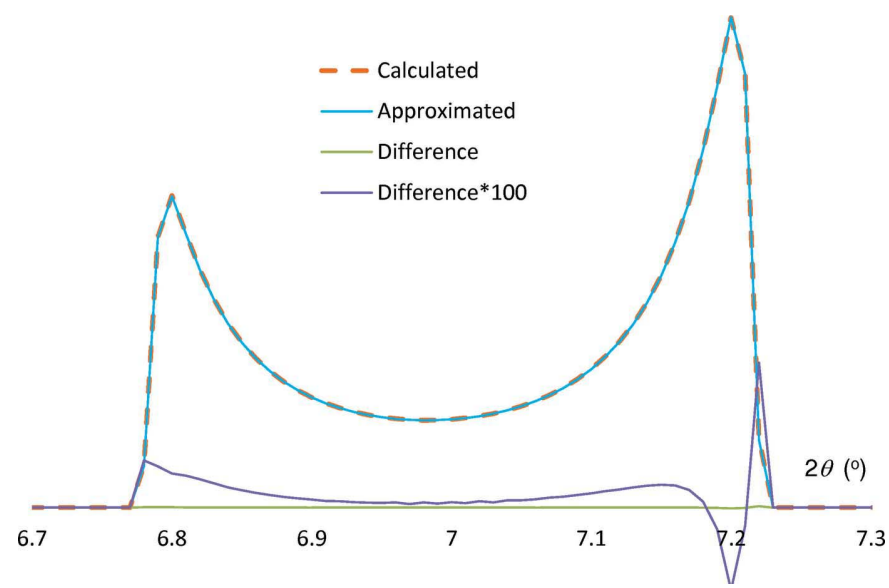

Figure 10

Convergent beam geometry disc aberration approximated at $7^{\circ} 2 \theta$ compared with calculated aberration. The calculated aberration uses the method described in the paper. The approximated aberration uses equation (9) with the $A_{1}$ and $A_{3}$ aberrations calculated using the method described in the paper, one at $5^{\circ} 2 \theta$ and one at $9^{\circ} 2 \theta . r=1 \mathrm{~mm}, R_{\mathrm{s}}=$ $200 \mathrm{~mm}, R_{\mathrm{f}}=800 \mathrm{~mm}$ and $\mu=20 \mathrm{~cm}^{-1}$.

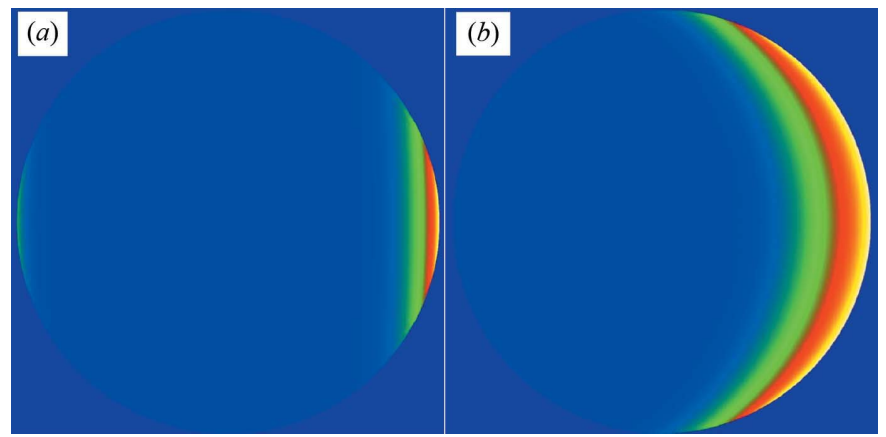

Figure 11

$I(x, y)$ for $\mathrm{LaB}_{6}$ with $R_{\mathrm{s}}=R_{\mathrm{f}}=200 \mathrm{~mm}$ and $r=0.5 \mathrm{~mm}$ at $(a) 20^{\circ} 2 \theta$ and (b) $120^{\circ} 2 \theta \cdot \mu=58 \mathrm{~cm}^{-1}$, similar to what can be expected with molybdenum $K \alpha$ radiation with a packing density of 0.4 . diameter of $0.3-0.5 \mathrm{~mm}$ and scanned with Stoe and Bruker AXS D8 diffractometers equipped with a focusing Johannsontype $\mathrm{Ge}(220)$ monochromator and using molybdenum $K \alpha_{1}$ radiation. Scans were in the range $5-90^{\circ} 2 \theta$ and $5-120^{\circ} 2 \theta$, respectively, and with step sizes in the data of 0.012 and $0.004^{\circ} 2 \theta$, respectively, with the diffractometers in convergent
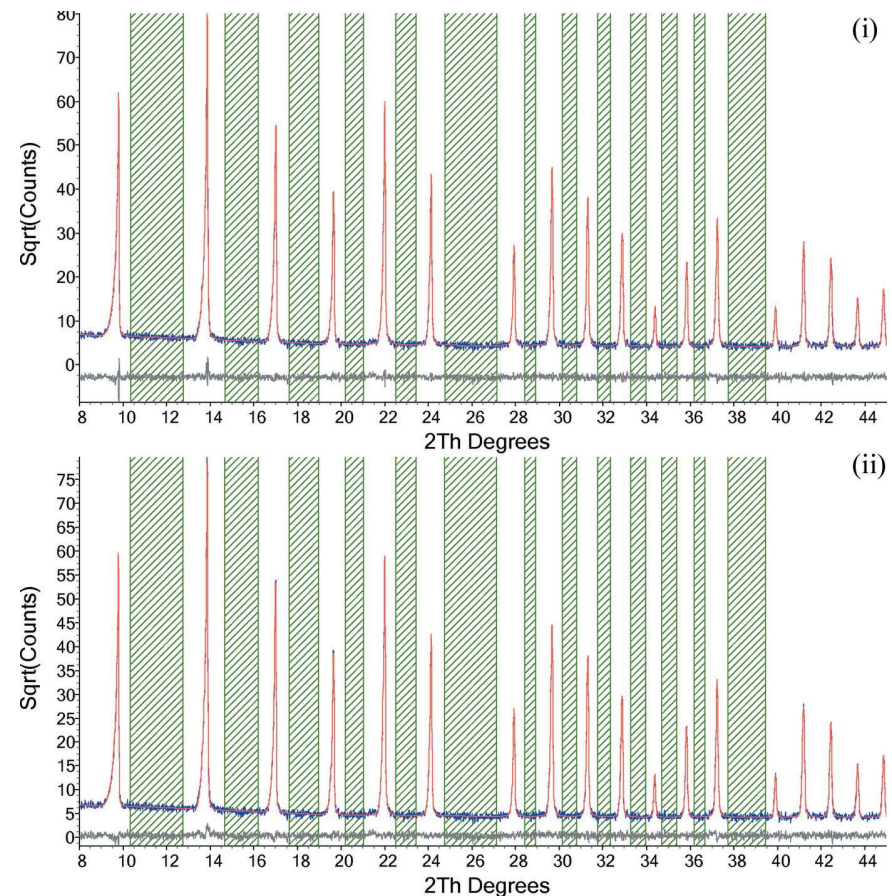

(a)
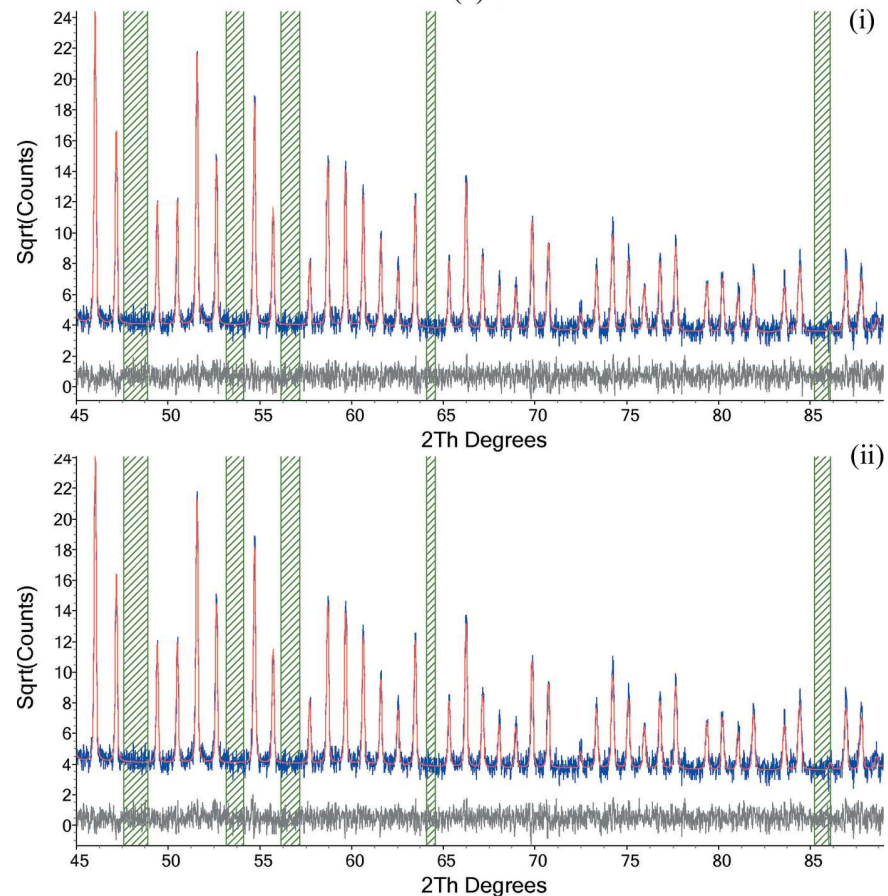

(b)

Figure 12

$\mathrm{LaB}_{6}$ refinement fit for (a) low-angle and (b) high-angle Stoe diffractometer data, obtained (i) empirically using $\tan (\theta)$ Gaussian broadening and (ii) using the present disc convolution. Green thatched regions correspond to regions that have been excluded from the refinement. 
Table 3

Convergent beam geometry $\mathrm{LaB}_{6}$ refinement results for Stoe and Bruker AXS diffractometers for cases (1) and (3), obtained empirically using $\tan (\theta)$ Gaussian broadening, and cases (2) and (4), using the present disc convolution.

Expected values are in bold. Underlined values were fixed and not refined. Equivalent temperature factors, $B_{\text {eq }}=8 \pi^{2} U_{\text {iso, }}$, in all cases were set to 0.5 . The space group used was $P m \overline{3} m$; fractional coordinates were not refined and were set to $(0,0,0)$ for the La site and $\left(0.19169, \frac{1}{2}, \frac{1}{2}\right)$ for the $B$ site.

\begin{tabular}{|c|c|c|c|c|}
\hline & \multicolumn{2}{|l|}{ Stoe } & \multicolumn{2}{|l|}{ Bruker AXS D8 } \\
\hline & Case (1) & Case (2) & Case (3) & Case (4) \\
\hline & Semi-empirical & Disc convolution & Semi-empirical & Disc convolution \\
\hline$R_{\mathrm{wp}}(\%)$ & 11.12 & 10.61 & 9.37 & 9.48 \\
\hline$R_{\text {Bragg }}(\%)$ & 2.28 & 2.23 & 2.28 & 2.29 \\
\hline Soller slit angle $\left({ }^{\circ}\right)$ & $4.29(3)$ & $4.30(3)$ & $2.818(8)$ & $2.842(9)$ \\
\hline Lattice parameter & $4.15499(9)$ & $4.15663(7)$ & $4.15604(7)$ & $4.15618(5)$ \\
\hline Zero error $\left({ }^{\circ} 2 \theta\right)$ & $-0.0045(23)$ & $-0.001(2)$ & $-0.0045(23)$ & $0.040(1)$ \\
\hline Perpendicular energy dispersive $(\mathrm{mm})$ & $-7.5(3)$ & $-0.43(36)$ & $-6.19(13)$ & $-5.81(10)$ \\
\hline Parallel energy dispersive (mm) & $1.1(0.5)$ & $-0.37(41)$ & $-5.46(28)$ & $-5.54(22)$ \\
\hline Gaussian $\times \tan (\theta)\left({ }^{\circ} 2 \theta\right)$ & $0.1741(8)$ & - & $0.0941(6)$ & - \\
\hline Sabine intensity & $1.42(2)$ & - & $1.014(10)$ & - \\
\hline Focal length $(\mathrm{mm})$ & - & $198(1), \mathbf{2 1 7}$ & - & $235(1), 217$ \\
\hline Capillary diameter $(\mathrm{mm})$ & - & $0.5718(1), \mathbf{0 . 3}-\mathbf{0 . 5}$ & _- & $0.350(2), \mathbf{0 . 3}-\mathbf{0 . 5}$ \\
\hline Packing density & - & $0.312(4), \mathbf{0 . 3 - 0 . 6}$ & - & $\underline{0.4}$ \\
\hline \multicolumn{5}{|c|}{ Refinement with perpendicular and parallel displacement parameters fixed and set to zero } \\
\hline$R_{\mathrm{wp}}(\%)$ & 11.92 & 11.43 & 10.62 & 10.72 \\
\hline Lattice parameter $(\AA)$ & $4.15694(2)$ & $4.15674(4)$ & $4.156541(8)$ & $4.156543(8)$ \\
\hline
\end{tabular}

Table 4

Percentage correlations obtained from the correlation matrix for $\mathrm{LaB}_{6}$ refinement of data from Stoe and Bruker AXS D8 instruments [cases (2) and (4) of Table 3].

Percentage correlation of the $a$ lattice parameter with $2 \theta$ shift parameters

\begin{tabular}{lll}
\hline & Stoe & Bruker AXS D8 \\
\hline Zero error & 49 & 89 \\
Perpendicular displacement & 56 & 72 \\
Parallel displacement & 44 & 90 \\
\hline
\end{tabular}

beam geometry and the focus of the beam approximately at the detector, or $R_{\mathrm{f}}=R_{\mathrm{s}}$. The receiving slit width was $0.1 \mathrm{~mm}$ and Soller slits were placed in the diffracted beam. The data were Rietveld refined using TOPAS-Academic for two scenarios: $(a)$ a semi-empirical approach applying Sabine $e t$ al. (1998) intensity corrections plus a Gaussian convolution that increases in width with $\tan (\theta)$; and $(b)$ the present capillary convolution (see Table 3). In both scenarios the perpendicular and parallel displacement of the diffractometer axis from the specimen axis was refined using the formulation of Scarlett $e t$ al. (2011). Regions of the diffraction pattern void of peaks were excluded from the refinement. The fits for both scenarios are good, with similar $R_{\text {wp }}$ and $R_{\text {Bragg }}$ values. The Gaussian broadening for the semi-empirical scenario, cases (1) and (3), is large; the Stoe diffractometer fit, case (1), has small peak position misfits on the low-angle 100 and 110 peaks at 9.8 and $13.8^{\circ} 2 \theta$, respectively, as shown in Fig. 12. These misfits are a result of there being too little broadening, which could be due to instrument misalignment factors. The D8 diffractometer is sharper with a smaller Soller acceptance angle and a smaller capillary diameter. The larger than expected parallel and perpendicular displacements for cases (1), (3) and (4) are probably due to the high correlations between the $a$ lattice parameter and the $2 \theta$ shift parameters, as seen in Table 4. These high correlations emphasize the need for careful instrument alignment with the displacement parameters fixed to zero and not refined. Doing so on these data, except for case (2), produces the significantly larger $R_{\mathrm{wp}}$ values seen at the bottom of Table 4. It is worth noting that negligible peak shifts are expected for this instrument and specimen configuration, where $\mu$ is expected to be $\sim 60 \mathrm{~cm}^{-1}$ and the focal length is expected to be similar to the diffractometer radius; from Table 1 $(a)$ the expected maximum centroid shift for $r=1 \mathrm{~mm}$ is $<0.001^{\circ} 2 \theta$; for $r<1 \mathrm{~mm}$ the expected shift should be even less.

Except for the large displacement parameters of case (4), the present disc convolution fits the whole $2 \theta$ range with parameters that are physically reasonable, as seen in Table 3, cases (2) and (4), and Fig. 12. The linear absorption coefficient used was that calculated for $\mathrm{LaB}_{6}$ and then multiplied by a refinable packing density parameter; this parameter refined to a reasonable value of 0.31 . The focal length, $R_{\mathrm{f}}$, refined to 198 and $235 \mathrm{~mm}$; this is not far off the perfectly focused beam of $217 \mathrm{~mm}$. The diameter of the capillary refined to reasonable values of 0.57 and $0.35 \mathrm{~mm}$. Fixing, and not refining, capillary diameters to accurate measured values would reduce correlations and improve robustness in the fitting process.

\section{Discussion}

The accuracy of the disc convolution presented here can be broken down into two parts; the intensity accuracy and the peak shape/position accuracy. The intensity accuracy is governed by the number of data points sampled across the disc, which is dependent on the chosen value for $N_{y}$. For a very small $\mu$, the intensity can be accurate using a small $N_{y}$. Too small a value of $N_{y}$, however, leads to inaccurate peak shapes and positions. For a sufficiently large $N_{y}$, equation (5) solves these problems, concentrating points in parts of the disc that contribute to intensity. $N_{y}=40$ results in very accurate aberrations with regard to intensity, shape and position, as shown in Tables 1 and 2. This accuracy has been measured against the disc aberration itself and does not include additional broadening as would be experienced with real observed data originating from the source emission profile, and instrument and specimen broadening. It is therefore probable that the extreme accuracy obtained with $N_{y}=40$ is not warranted in most situations. However, the speed at which the disc aberration can be calculated is small in comparison to other 
calculations necessary in synthesizing line profiles, and hence reduction in $N_{y}$ is not warranted.

\section{Conclusion}

A fast and accurate numerical approach to calculating aberrations originating from a capillary in the equatorial plane, a disc, has been developed for convergent, divergent and parallel beam instrument geometries. The aberration is then convoluted with an emission profile and instrument and specimen aberrations to form a diffraction pattern, which is used in Rietveld refinement. Refinement of the fundamental parameters of the capillary diameter, the focal length and the specimen linear absorption coefficient should agree with expected or measured values.

\section{APPENDIX $A$}

\section{A1. Validation of model, ray tracing}

A ray-tracing model was constructed to simulate the effect of convergent, divergent and parallel monochromatic incident $\mathrm{X}$-ray beams of uniform intensity on the resultant aberration peak shapes, positions and intensities. In this model (Fig. 13), the X-ray beam is incident on the disc from left to right, along the $x$ axis, and completely bathes the disc. There are $N$ points, $O^{\prime}$, distributed evenly throughout the disc. Each ray enters and exits the disc at $R$ and $R^{\prime}$, diffracting at $O^{\prime}$ through an angle $2 \theta$, and is detected at $D$, at an angle $2 \varphi$. The focal length and specimen-to-detector distance are given by $R_{\mathrm{f}}$ and $R_{\mathrm{s}}$, respectively. The total path length through the disc, $P$, is given by $P_{\mathrm{p}}+P_{\mathrm{s}}$. To form a ray-tracing aberration, $N$ points were generated in polar coordinates

$$
(\rho, \kappa)=\left(r U_{1}^{1 / 2}, 360 U_{2}\right),
$$

where $U$ is a random number uniformly distributed in the range $[0,1)$. The square root ensures that each point has an equal area associated with it. The calculation of the angle $\alpha$ allows for the easy conversion of the model between convergent, divergent and parallel incident $\mathrm{X}$-ray beams. $\alpha$ is given by

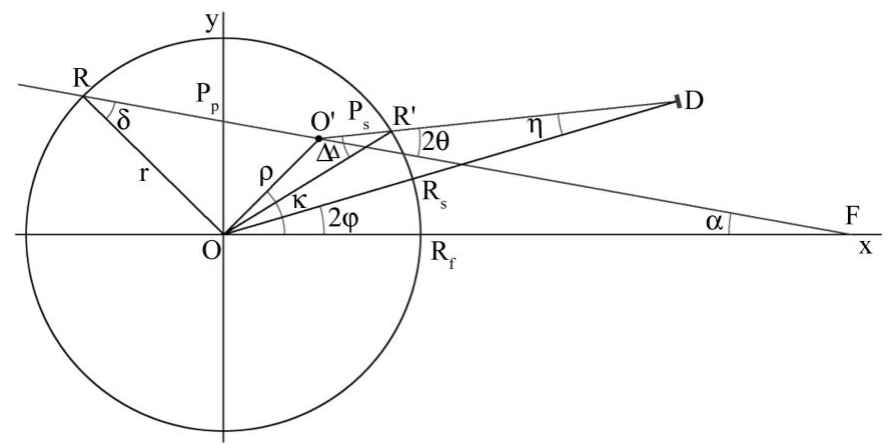

Figure 13

Ray-trace geometry. A ray, focused at $\mathrm{F}$, enters and exits the disc at $R$ and $R^{\prime}$, diffracting at $O^{\prime}$ through an angle $2 \theta$, and is detected at $D$ at an angle $2 \varphi$, having travelled through the disc with a total path length of $P_{\mathrm{p}}+P_{\mathrm{s}}$.

$$
\alpha=G \arctan \left[\frac{\rho \sin (\kappa)}{R_{\mathrm{f}}-G \rho \cos (\kappa)}\right],
$$

where $G$ denotes the instrument geometry; 1 is convergent, -1 is divergent and 0 is parallel. The path length can then be calculated by

$$
\begin{gathered}
\delta=\arcsin \left[\frac{\rho \sin (\kappa+\alpha)}{r}\right], \\
\Delta=\arcsin \left[\frac{\rho \sin (\kappa+\alpha-2 \theta)}{r}\right],
\end{gathered}
$$

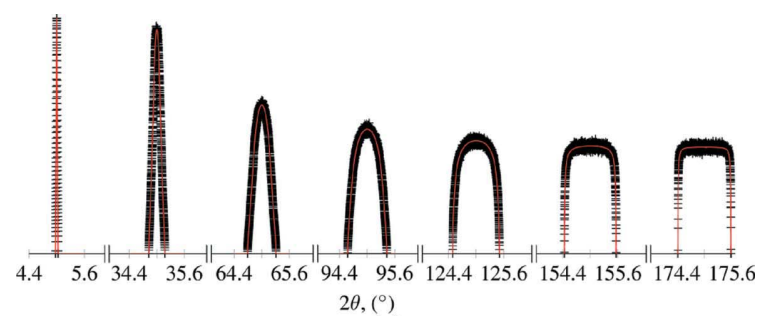

(a)

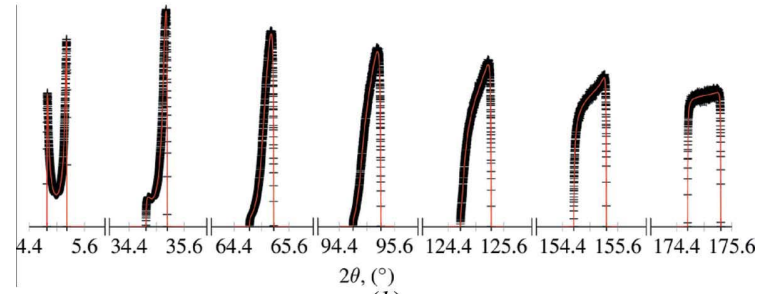

(b)

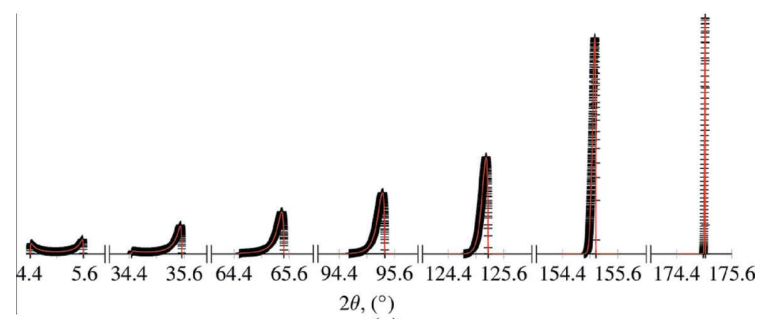

(c)

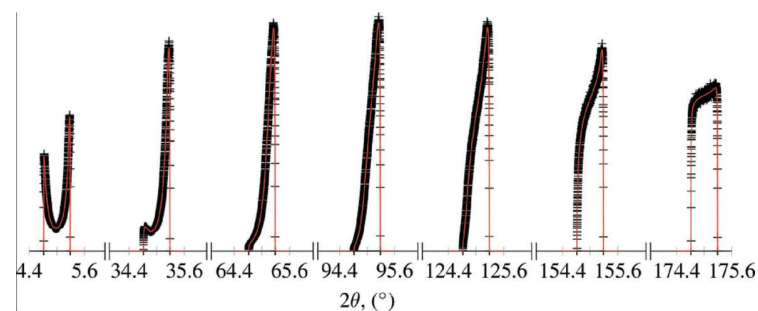

(d)

Figure 14

Ray-tracing and aberration model fits for $(a)$ convergent $\left(R_{\mathrm{f}}=200 \mathrm{~mm}\right.$, $\left.R_{\mathrm{p}}=1.33 \%\right),(b)$ semi-convergent $\left(R_{\mathrm{f}}=800 \mathrm{~mm}, R_{\mathrm{p}}=1.07 \%\right),(c)$ divergent $\left(R_{\mathrm{f}}=200 \mathrm{~mm}, R_{\mathrm{p}}=0.835 \%\right)$ and $(d)$ parallel $\left(R_{\mathrm{p}}=0.989 \%\right)$ incident beams. The ray-tracing data were calculated for $R_{\mathrm{s}}=200 \mathrm{~mm}, r=$ $1 \mathrm{~mm}, \mu=20 \mathrm{~cm}^{-1}, N=20 \times 10^{6}$ and $\varepsilon_{\Delta}=0.0005^{\circ}$. Only a subset of the peaks are shown for clarity. All data within each section are displayed on a common vertical axis, except for the first peak in $(a)$, where the vertical axis is compressed by a factor of 6 , and the last peak in $(c)$, where the factor is 4.5 . 


$$
P=P_{\mathrm{p}}+P_{\mathrm{s}}=\rho\left[\frac{\sin (\kappa+\alpha+\delta)}{\sin (\delta)}+\frac{\sin (\kappa+\alpha-\Delta-2 \theta)}{\sin (\Delta)}\right]
$$

where $2 \theta$ is the diffraction angle taken with respect to the incident ray. The relative transmitted intensity can then be calculated as

$$
t=\exp (-\mu P)
$$

where $\mu$ is the linear absorption coefficient of the diffracting material. The diffracted ray is detected at $2 \varphi$, where this angle is taken with respect to the centre of the goniometer. The deviation of the diffracted beam from its expected position, $\varepsilon=2 \varphi-2 \theta$, can be calculated by

$$
\begin{gathered}
\eta=\rho \sin \left(\frac{\kappa+\alpha-2 \theta}{R_{\mathrm{s}}}\right), \\
\varepsilon=\eta-\alpha .
\end{gathered}
$$

These equations allow for a complete mapping of $(\rho, \kappa) \rightarrow(2 \varphi, t)$. To calculate the entire diffraction peak shape and intensity, centred $2 \varphi$ bins of width $\varepsilon_{\Delta}$ were created and a total relative transmitted intensity for each bin was calculated as

$$
T=\frac{\sum t}{N \varepsilon_{\Delta}},
$$

where the sum was taken over all values of $t$ for which the corresponding value of $2 \varphi$ falls in that particular bin.

\section{A2. Comparison with disc aberration}

A total of $20 \times 10^{6}$ points were evenly distributed randomly throughout the disc, and the relative transmitted intensity, $\exp (-\mu \mathrm{P})$, and $2 \varphi$ were calculated for each point. The relative transmitted intensities were then placed into centred $2 \varphi$ bins of width $0.0005^{\circ}$, giving the total relative diffracted intensity for a given diffraction angle, $2 \theta$. Diffraction peaks were generated every $5^{\circ}$ from 5 to $175^{\circ} 2 \theta$. Numerous combinations of $R_{\mathrm{s}}, R_{\mathrm{f}}, r$ and $\mu$ were tested. Examples of $(a)$ convergent $\left(R_{\mathrm{f}}=\right.$ $200 \mathrm{~mm}),(b)$ semi-convergent $\left(R_{\mathrm{f}}=800 \mathrm{~mm}\right),(c)$ divergent $\left(R_{\mathrm{f}}=200 \mathrm{~mm}\right)$ and $(d)$ parallel incident beams for $R_{\mathrm{s}}=$ $200 \mathrm{~mm}, r=1 \mathrm{~mm}$ and $\mu=20 \mathrm{~cm}^{-1}$ are shown in Fig. 14. The profile $R$ factors,

$$
R_{\mathrm{p}}=\left(\frac{\sum_{m}\left|Y_{\mathrm{o}, m}-Y_{\mathrm{c}, m}\right|}{\sum_{m} Y_{\mathrm{o}, m}}\right)^{1 / 2}
$$

for the various fits are given in the figure caption. $Y_{\mathrm{o}}$ are the observed intensities, and $Y_{\mathrm{c}}$ are the calculated intensities.

\section{Acknowledgements}

The authors would like to thank Dr Robert Dinnebier of the Max Planck Institute Stuttgart for supplying $\mathrm{LaB}_{6}$ data for use in this paper.

\section{References}

Cheary, R. W. \& Coelho, A. A. (1998). J. Appl. Cryst. 31, 851-861.

Coelho, A. A. (2017). TOPAS-Academic V7, http://www.topasacademic.net/.

Coelho, A. A., Chater, P. A. \& Kern, A. (2015). J. Appl. Cryst. 48, 869-875.

Dwiggins, C. W. (1972). Acta Cryst. A28, 219-220.

Dwiggins, C. W. (1975). Acta Cryst. A31, 146-148.

NIST (2000). SRM 660a. National Institute of Standards and Technology, US Department of Commerce, Gaithersburg, MD, USA.

Rietveld, H. M. (1969). J. Appl. Cryst. 2, 65-71.

Sabine, T. M., Hunter, B. A., Sabine, W. R. \& Ball, C. J. (1998). J. Appl. Cryst. 31, 47-51.

Scarlett, N. V. Y., Rowles, M. R., Wallwork, K. S. \& Madsen, I. C. (2011). J. Appl. Cryst. 44, 60-64.

Sulyanov, S., Gogin, A. \& Boysen, H. (2012). J. Appl. Cryst. 45, 93-97. 